\title{
Labyrinthe
}

$35 \mid 2010$ (2)

Empire Reader

\section{«Colonialisme » et « Empires coloniaux »}

\section{Jürgen Osterhammel}

Traducteur : Thierry Carpent

\section{OpenEdition \\ Journals}

Édition électronique

URL : http://journals.openedition.org/labyrinthe/4083

DOI : $10.4000 /$ labyrinthe.4083

ISSN : 1950-6031

Éditeur

Hermann

Édition imprimée

Date de publication : 28 août 2010

Pagination : 57-68

ISBN : 9782705669973

Référence électronique

Jürgen Osterhammel, « « Colonialisme » et « Empires coloniaux » », Labyrinthe [En ligne], 35 | 2010 (2), mis en ligne le 27 juillet 2012, consulté le 10 décembre 2020. URL : http://journals.openedition.org/ labyrinthe/4083 ; DOI : https://doi.org/10.4000/labyrinthe.4083

Propriété intellectuelle 


\title{
«Colonialisme » et « Empires coloniaux »
}

\author{
Jürgen OSTERHAMmEL \\ Contact : Juergen.Osterhammel@uni-konstanz.de
}

(C 1995 C. H. Beck Verlag. La version originale de ce texte est parue dans Kolonialismus : Geschichte, Formen, Folgen, Munich, C. H. Beck, 1995 [6e éd. revue, 2009] , p. 19-28. Reproduction sur autorisation. Version française revue et corrigée par l'auteur.

\section{Le colonialisme : une définition}

Qu'est-ce donc que le colonialisme ? Comment peut-on définir ce concept indépendamment de celui de colonie ? L'historien Philip Curtin, spécialiste de l'Afrique, parle de façon générale de «domination par un peuple issu d'une autre culture ${ }^{1} \gg$. Cette formulation contient deux éléments décisifs : la «domination » et «l'appartenance à une culture étrangère ». Mais elle doit être précisée. Historiquement, ceux qui ont subi la domination exercée par des étrangers n'ont pas toujours considéré que celle-ci fût illégitime. L'Égypte, province de l'Empire ottoman de 1517 à 1798, a ainsi été placée sous la domination totalement interventionniste des Turcs sans que cela empêche la reconnaissance généralisée du système par la population locale arabophone ${ }^{2}$. Dans ce cas, la communauté de religion et, partant, la reconnaissance unanime du caractère d'obligation que revêtait la conception islamique du gouvernement juste ont compensé la différence linguistique.

Pour dégager plus précisément la spécificité historique du colonialisme de l'époque moderne (on pourrait peut-être parler ici de colonialisme moderne au sens courant du terme), il convient de compléter la formule fondamentale de Curtin par les trois éléments suivants. Premièrement, le colonialisme n'est pas une relation quelconque entre maîtres et esclaves, mais un rapport dans lequel une société tout entière est dépossédée de son développement historique propre pour être dirigée par des étrangers, et ce en fonction des besoins et des intérêts (essentiellement économiques) des maîtres coloniaux. Pour le cadre théorique de la définition concep- 
"Les différences entre les évaluations coloniales et postcoloniales sont [...] très grandes. Cependant, une analogie subsiste : que l'on considère le colonialisme comme porteur de civilisation ou comme une forme d'abus et la cause des problèmes au sein des anciennes colonies, dans les deux cas chacun part du principe que l'influence du colonialisme a été considérable. On pourrait parler d'une école maximaliste. Néanmoins il existe également, notamment dans l'historiographie asiatique, une école minimaliste qui estime que l'influence de l'expansion occidentale sur les civilisations asiatiques séculaires est extrêmement réduite. Ces civilisations présentaient une plus grande stabilité et étaient plus impénétrables qu'on l'avait imaginé. "Un château de sable", c'est ainsi qu'on a pu résumer un jour le résultat de plusieurs siècles de colonialisme néerlandais en Indonésie. Nul historien ne défend encore la thèse que les peuples d'outre-mer ne furent que les victimes apathiques des maîtres coloniaux. On souligne aujourd'hui fortement combien les puissances coloniales européennes, au plus fort de l'âge d'or du colonialisme, restèrent dépendantes de la collaboration des peuples colonisés ».

Hendrik Lodewijk [Henri] Wesseling, Les Empires coloniaux européens, 1815-1919 (trad. du néerlandais par Patrick Grilli), Paris, Gallimard, 2009 [2003], préface à l'édition française, p. 13.

tuelle, il est dans un premier temps accessoire que, dans la pratique, les gouvernements coloniaux aient rarement atteint un but aussi ambitieux et que les moyens leur aient souvent manqué pour le réaliser. Le colonialisme moderne repose sur la volonté de mettre des sociétés «périphériques » au service des «métropoles ».

Deuxièmement, la manière dont colonisateurs et colonisés restent étrangers les uns aux autres est très significative. Le refus volontaire, chez ces nouveaux maîtres, d'aller à la rencontre de la culture des sociétés qu'ils ont soumises, est une caractéristique du colonialisme moderne dont l'histoire universelle offre peu d'exemples. Nulle part l'expansion européenne n'a donné lieu à une synthèse culturelle de type " hellénistique ». On attendait des colonisés une acculturation les amenant à adopter pour une large part les valeurs et coutumes européennes sans que - à quelques exceptions près dans l'empire portugais - on puisse relever de la part des colonisateurs une réelle contre-acculturation donnant lieu à l'adoption d'éléments des civilisations dominées. L'impossibilité de tels rapprochements a été justifiée au XIX ${ }^{\mathrm{e}}$ siècle par l'existence de hiérarchies "raciales » prétendument insurmontables. Une définition du colonialisme doit prendre en compte le refus de s'adapter dont les colonisateurs ont fait preuve. 
Enfin, le troisième point est étroitement lié au second. Le colonialisme moderne n'est pas seulement une relation de domination dont la description relève de l'histoire des structures, mais dans le même temps une interprétation particulière de ce rapport. Une mentalité spécifique fait partie de son essence même, au point qu'on a pu dire à ce sujet qu'il s'agissait d'une « formation idéo$\operatorname{logique}^{3} »$. Depuis les théoriciens ibériques et anglais du $\mathrm{XVI}^{\mathrm{e}}$ siècle, l'expansion européenne a été glorifiée comme l'accomplissement d'une mission universelle : elle participait du plan divin du salut induisant la mission auprès des
" L'empire, tel qu'on peut le déduire du mécanisme théorique d'Ibn Khaldûn, est autiste. II ne parvient pas à penser d'autre réalité que lui et sa négation, c'est-à-dire le sauvage. L'empire partage avec la tribu néolithique chère à Lévi-Strauss l'idée que l'humanité se résume à lui. La Rome des Antonins, la Chine des Tang, I'Islam de l'apogée des Abbassides sont des empires en ce sens qu'ils n'ont pas de frontières avec des entités qui leur seraient comparables, mais des confins - ou des murailles ; l'empire n'a pas de voisins mais des Barbares, ce qu'lbn Khaldûn nomme des bédouins, et qu'il oppose aux sédentaires sujets de l'empire. Cette réduction du sens, et d'abord de l'histoire, à une seule construction politique, au-delà de laquelle s'étend le monde de l'informe et de l'impensable, me paraît être une des caractéristiques de l'empire ».

Gabriel Martinez-Gros, « L'empire et son espace. Conclusion », Hypothèses 2007/1, p. 275-276.

païens ; elle relevait d'un mandat laïc reçu en vue de «civiliser» les « barbares » ou les «sauvages »; il s'agissait d'un privilège lourd à porter, d'un «fardeau de l'homme blanc», etc. Ce point de vue se fondait sur la conviction que la culture des colonisateurs jouissait d'une suprématie culturelle. Aussi bien le colonialisme des États-Unis que celui du Japon se sont eux aussi largement servi de ce genre de rhétorique reposant sur l'idéologie missionnaire. Des cultures traditionnelles, comme celle de la Chine, considéraient comme une évidence leur caractère de modèles indépassables sans pour autant l'imposer à leurs voisins. C'est uniquement dans le colonialisme moderne qu'un tel orgueil ethnocentrique a pris un tour aussi agressivement expansionniste et que le plus grand nombre a été soumis à un « joug spirituel » par un petit nombre ${ }^{4}$. Il en résulte que la caractérisation des structures de dépendance colonialistes est insuffisante si elle ne prend pas en compte « l'esprit du colonialisme » qui les anime. Du reste, un tel état d'esprit (ou dévoiement de l'esprit) a survécu à l'époque coloniale. 
On peut donc donner la définition suivante :

Le colonialisme est une relation de domination établie entre des collectivités dans laquelle les décisions fondamentales concernant le mode de vie des colonisés sont prises par une minorité de colonisateurs issus d'une culture différente et rétifs à toute adaptation ; ces derniers prennent ces décisions et les appliquent effectivement en accordant la priorité à des intérêts extérieurs à ceux des colonisés. Au cours des temps modernes, le colonialisme est lié à des doctrines de justification relevant d'une idéologie missionnaire, et qui reposent sur la conviction, de la part des colonisateurs, d'être culturellement supérieurs.

La tentative consistant à ne pas faire dériver le «colonialisme » de la notion, définie préalablement ${ }^{5}$, de « colonie », mais de le définir indépendamment de celle-ci, amène obligatoirement à poser la question du rapport existant entre ces deux phénomènes. La colonie et le colonialisme vont normalement de pair, mais il y a cependant des cas-limites démontrant qu'il n'y a pas toujours adéquation entre eux. Le premier est celui de colonies sans colonialisme, qu'on trouve là où, au sein des sociétés coloniales, il n'y avait pas de population autochtone majoritaire et où ces sociétés étaient donc homogènes par leur «blancheur». Les contemporains ont aussi considéré ces colonies comme des «États planteurs » de pays européens établis dans des pays « vides ». Cela vaut essentiellement pour les colonies de peuplement du type de la « NouvelleAngleterre ». Pratiquant dans de vastes proportions une colonisation (dite de frontière), celles-ci défrichaient des terres «sauvages » intérieures en vue de leur exploitation agricole et anéantissaient du même coup l'espace vital des chasseurs et bergers autochtones. Du fait de l'absence de sujets « indigènes », le rapport de domination caractérisant le colonialisme n'a cependant pas pu s'y développer. Ces sociétés n'ont donc pas non plus été « décolonisées » par l'expropriation et l'expulsion des colonisateurs, comme cela a été le cas par exemple en Algérie. Elles ont obtenu leur indépendance et sont devenues des États nationaux à la suite d'une sécession qu'une révolution a rendue abrupte (les treize colonies formant par la suite les États-Unis) ou bien par un phénomène d'éloignement progressif et largement consensuel du centre de l'empire situé en Europe (Canada, Australie). 
À l'autre bout du spectre, on doit prendre en compte le colonialisme sans colonies, c'est-à-dire des situations dans lesquelles la relation de dépendance «coloniale » n'est pas établie entre une «métropole » et une colonie éloignée de celle-ci dans l'espace, mais entre des « centres » dominants et des «périphéries » dépendantes se situant à l'intérieur d'États nationaux ou d'empires territoriaux cohérents. Face à de tels cas, on a tenté de résoudre la difficulté en forgeant la construction théorique $\mathrm{du}$ « colonialisme interne » qui prend essentiellement comme exemple la relation entre l'Angleterre et la « périphérie celtique » des Iles britanniques (Pays de Galles, Écosse, Irlande).

On peut se demander si une telle lecture ne force pas le concept de colonialisme de façon métaphorique ${ }^{6}$. La représentation de relations sous-coloniales à l'intérieur d'empires coloniaux organisés hiérarchiquement pose moins de problèmes : le rapport primaire colonialiste est alors établi non pas entre une métropole et une colonie, mais entre

"L'usage métaphorique du terme empire, que ce soit pour signifier un pouvoir d'État sans limites ou des initiatives de réforme et d'amélioration, tend à brouiller les catégories dont nous avons besoin pour mener à bien l'analyse des formes politiques dans leur spécificité ».

Craig Calhoun, Frederick Cooper, Kevin W. Moore (dir.), Lessons of Empire. Imperial Histories and American Power, New York, The New Press, 2006, « Introduction », p. 2. cette colonie et une autre colonie de l'empire. Ainsi, à certaines époques, on peut considérer que l'Angola était une sous-colonie du Brésil, tout comme les Philippines étaient pour ainsi dire une succursale du Mexique. L'Inde était entourée d'une couronne de satellites : si pendant les années 1920, les colons blancs n'ont pas pris le pouvoir dans la colonie de peuplement qu'était alors le Kenya comme ils l'ont fait en Rhodésie du sud, c'est parce que le gouvernement des Indes britanniques protégeait les populations indiennes ayant émigré en Afrique de l'est.

\section{Empires coloniaux et « empire informel »}

Au cours des temps modernes, la plupart des colonies étaient intégrées à des empires coloniaux. L'idée d'empire inclut le fait que plusieurs périphéries subordonnées en étoile au centre de l'empire entretiennent 
des relations entre elles, même si ces dernières sont plus faibles que les relations que chacune d'entre elles entretient avec le centre. Dans certains cas, la « masse critique » de l'empire colonial ne suffit pas pour que l'on puisse parler d'empire. La Belgique ne contrôlait que deux colonies : le Congo belge (actuel Zaïre) de 1885 à 1960 et le Rwanda et le Burundi de 1916 à 1962. Les seules colonies territoriales des États-Unis étaient les Philippines (1898-1946) et Porto-Rico (1898-1952). Du fait de l'indépendance des États latino-américains, puis de la défaite subie lors de la guerre hispano-américaine de 1898, l'empire colonial espagnol, qui englobait autrefois de larges parties du monde, s'est trouvé réduit à une poignée de possessions minuscules en Afrique du Nord, si bien qu'au $\mathrm{Xx}^{\mathrm{e}}$ siècle, on ne peut plus parler d' " empire » espagnol. Dans tous ces cas, nous avons affaire à des possessions coloniales sans empire colonial. Il est possible d'en dire autant de l'empire des Pays-Bas qui, après la perte de Ceylan (1796) et du Cap de Bonne-Espérance (1794/1806), s'est réduit, outre le Surinam, aux Indes néerlandaises (Indonésie) — mais il s'agissait là d'une colonie fortement peuplée et d'une importance peu commune du point de vue économique pour la métropole.

Les empires européens d'outre-mer constitués pendant la période moderne étaient essentiellement et presque exclusivement des empires coloniaux. Leur situation changea à partir de la fin du XVIII siècle avec la distance qui s'est alors instaurée sur le plan des capacités économiques entre les économies européennes fondées sur la croissance et le monde d'outre-mer, avec l'intensification des relations économiques mondiales, l'amélioration de moyens militaires susceptibles d'être déployés partout dans le monde, et l'apparition d'une pensée politique aux dimensions stratégiques mondiales. C'est avant tout la Grande-Bretagne, à laquelle son économie et sa flotte ont permis d'exercer entre 1815 et 1880 le leadership mondial, qui, outre l'acquisition de colonies, a vu s'ouvrir un éventail varié d'autres possibilités susceptibles de faire valoir ses intérêts économiques et stratégiques. À bien des égards, il suffisait d'amener des États d'outre-mer restés politiquement autonomes (les États latino-américains, la Chine, le Japon, la Thaïlande, la Perse, l'Empire ottoman, etc.) à ouvrir leurs marchés aux produits de l'industrie britannique et à garantir la propriété étrangère de jure et de facto. C'est à ces fins qu'ont servi les pressions diplomatiques, les menaces militaires, voire des interventions ponctuelles de la marine de guerre comme ce fut le cas lors de la « guerre de l'opium », grâce à laquelle l'Empire chinois fut « ouvert » en 1842. 
Dans tous ces cas, une domination coloniale aurait été trop coûteuse et aurait entraîné des responsabilités politiques inutiles. On laissait donc la charge du gouvernement aux souverains autochtones anglophiles, lesquels étaient toutefois rarement des « collaborateurs » enthousiastes.

Cette distinction entre sauvegarde « formelle » et « informelle» de ses intérêts face à des pays militairement plus faibles et économiquement « attardés » (comme on disait alors), entre « empire formel » et « empire informel ${ }^{7}$ » ne s'applique pas uniquement à l'empire britannique, mais peut, dans une large mesure, être étendue au moins aux États-Unis et au Japon. Cuba, par exemple, est un exemple classique de ce qu'est un « empire informel »: cette ancienne colonie espagnole (jusqu'en 1898) a été de 1902 jusqu'à la prise du pouvoir par Fidel Castro en 1959 une république officiellement indépendante ; toute son économie ne s'en trouvait pas moins aux mains des Américains, et le gouvernement des États-Unis intervenait à divers titres dans sa politique intérieure. Cuba était donc un territoire semi-souverain exploité par les États-Unis. Les Japonais, dont l' «empire formel » était constitué principalement des colonies extrêmement rentables qu'étaient Taïwan (1895-1945), la Corée (1910-1945) et l'État fantoche du Mandchoukouo (composé à partir des trois provinces du nord-est de la Chine, de facto une colonie de 1931 à 1945), l'ont complété en établissant en Chine à partir de 1905 une zone d'influence qu'ils ont progressivement étendue ${ }^{8}$. La politique de l'Empire allemand a également consisté avant 1914 à construire un « empire informel » en Chine, en Amérique latine et dans l'Empire ottoman.

Pour résumer, on peut différencier pour les $\mathrm{XIX}^{\mathrm{e}}$ et $\mathrm{XX}^{\mathrm{e}}$ siècles trois degrés de la sauvegarde des intérêts des « grands frères » face aux «petits frères »:

(1) La domination coloniale (= empire formel) : des étrangers remplacent les autorités locales (un gouverneur britannique prend par exemple la place d'un sultan malais). L'ordre politique précolonial cesse d'exister ou du moins de fonctionner librement. Ce sont des représentants de la puissance coloniale qui exercent les fonctions souveraines telles que l'imposition, la justice, les pouvoirs militaire et de police. Les relations étrangères des colonisés sont dans leur ensemble monopolisées par la puissance coloniale. Les définitions suggérées ci-dessus de ce que sont une « colonie » et le « colonialisme » s'appliquent pleinement.

(2) Le contrôle quasi colonial (= empire informel). L'État le plus faible demeure une structure de droit public autonome dotée d'un système 
politique propre. Il peut mener une politique étrangère et régler lui-même les affaires intérieures courantes. Il n'y a pas d'administration coloniale, mais parfois — et plus particulièrement dans le domaine financier — des autorités mixtes composées d'étrangers et d'indigènes (comme l'office maritime des douanes dans l'Empire chinois). Cependant, la souveraineté de l'État le plus faible est limitée. En exerçant des pressions de façon ponctuelle la (« diplomatie de la canonnière »), le « grand frère » a réussi à obtenir des privilèges garantis par des « traités inégaux » qui permettent la plupart du temps de soustraire, par le biais d'une juridiction consulaire et de l'exterritorialité, les ressortissants étrangers aux lois autochtones, de mettre en place un régime de libre échange (droits peu élevés à l'importation dans le cas où il n'y pas de souveraineté douanière), de stationner des troupes étrangères dans les eaux territoriales et sur des bases terrestres. Le « grand frère » est représenté par des consuls, des diplomates ou des « résidents » qui interviennent dans la politique intérieure pour « prodiguer des conseils », particulièrement lors des luttes de succession, et qui le cas échéant donnent du poids à leurs « conseils » en menaçant d'une intervention militaire. Dans les cas extrêmes, les dignitaires autochtones sont des fantoches, mais ils disposent normalement d'une certaine marge de manœuvre, qui peut même être considérable. L' « élite collaborationniste » idéale sur laquelle repose immanquablement l'empire informel doit disposer à l'intérieur d'une légitimité et d'une capacité à s'imposer suffisantes pour agir de façon efficace dans l'intérêt du « grand frère ». Les motivations de l'empire informel sont rarement stratégiques ou fondées sur des points de vue de prestige. La plupart du temps, il s'agit d'un moyen permettant d'assurer la sauvegarde d'intérêts économiques souvent importants (commerce, investissements directs, emprunts) qui se sont constitués sans soutien politique. De façon différente du colonialisme (empire formel), l'empire informel suppose une supériorité économique nettement considérable de la part du grand frère : il doit exister un potentiel permettant de «pénétrer » l'économie d'un pays d'outre-mer. Alors que, dans le cas de l'empire formel, la domination coloniale sur un seul et même territoire est indivisible et exclusive, il peut y avoir dans le cas de l'empire informel plusieurs «grands frères » qui s'accordent sur le principe de la « porte ouverte » offrant des chances égales pour tous ou sur la délimitation de « sphères d'influence » nationales.

(3) L'influence non-coloniale "déterminante » : dans ce cas-là, il n'y a entre le «grand frère » et le «petit frère » aucun rapport de 
domination. Il n'y a pas non plus de droits exceptionnels codifiés dans des «traités inégaux ». La supériorité économique du plus fort des deux États partenaires ou de ses institutions économiques de droit privé (par exemple des sociétés multinationales) et/ou sa fonction militaire de protection lui confèrent des possibilités d'influer sur la politique de l'autre État dont ne disposent pas les voisins «naturels » de celui-ci. Il s'agit d'un modèle de relation typique de l'asymétrie internationale à l'époque postcoloniale.

\section{Impérialisme}

Leur rayon d'action politique et économique a permis à l'Empire britannique des $\mathrm{XIX}^{\mathrm{e}}$ et $\mathrm{XX} \mathrm{x}^{\mathrm{e}}$ siècles et à quelques autres empires modernes de dépasser leur composante coloniale au sens strict. Dans quelques parties du monde, ils ont exercé une domination coloniale formelle, dans d'autres, ils se sont contentés de possibilités informelles d'intervention et de la sauvegarde de leurs intérêts. Il leur suffisait parfois d'être des voisins puissants. Ce qui était décisif, c'était d'obtenir par d'autres moyens des résultats la plupart du temps analogues. Que le pays d'outre-mer soit une colonie ou un État quasi colonial doté d'une souveraineté nominale et devant laisser prospérer librement les intérêts économiques étrangers sans avoir lui-même aucune influence sur les décisions économiques essentielles tant intérieures qu' extérieures, cela n'avait en règle générale aucune incidence pratique sur les conséquences économiques d'une présence étrangère dans ce pays.

L' « impérialisme » est le concept qui résume toutes les forces et les activités contribuant à la mise en place et au maintien de tels empires transcoloniaux. Ce qui fait partie de l'impérialisme, c'est aussi la volonté et la capacité d'un centre impérial de définir constamment comme impériaux ses intérêts nationaux et à les faire valoir universellement dans l'anarchie du système international. L'impérialisme implique donc non seulement une politique coloniale, mais une politique à l'échelle mondiale («Weltpolitik»), dans laquelle les colonies ne sont pas des buts en soi, mais des gages dans des enjeux globaux de pouvoir. L'idée en vogue à partir du dernier tiers du XIX ${ }^{\mathrm{e}}$ siècle dans les grandes puissances consistait à considérer que — par le jeu d'échanges (par exemple Héligoland contre Zanzibar), par la reconnaissance de prétentions géopoli- 
«Les Indiens ne connaissent l'empire que trop bien. De ce fait, quand nous considérons la domination des États-Unis dans le monde aujourd'hui, la plupart d'entre nous n'ont guère de mal à y reconnaître un empire. Il est pourtant nécessaire de comprendre que l'empire états-unien actuel, comparé aux empires anciens, est de nature fondamentalement différente. [...] Les dix dernières années du $x x^{e}$ siècle ont tout changé. [...] C'est un empire qui ne conquiert plus de territoire et n'impose plus sa propre administration ou sa propre fiscalité au pays vaincu. II n'envoie même plus ses forces armées combattre, à moins que cela ne soit rigoureusement nécessaire. C'est un empire démocratique. II reconnaît la souveraineté du peuple. Peut-on dire alors que le peuple d'un pays exerce une souveraineté sur le peuple d'un autre pays ? Cela correspondrait à la définition du colonialisme démodé du XIXe siècle, et il n'y aurait là rien de nouveau. Mais la réalité est autre. Lorsque des milliers de missiles ont décollé des navires états-uniens pour réduire en cendres la ville de Belgrade, personne n'a pensé que le peuple des États-Unis allait proclamer sa souveraineté sur le peuple de Serbie. [...] La préoccupation première des États-Unis était en réalité de ramener les troupes le plus vite possible au pays. Ce nouvel empire n'a pas de rival. Il est mondial. Sous son règne, aucun État n'exerce de souveraineté sur un autre. C'estl'empire qui est souverain. [...] La clef de l'empire n'est pas la force, mais le contrôle. S'il y a toujours une limite à la force, le contrôle n'en connaît aucune. La vision de l'empire est ainsi celle d'une démocratie mondiale ».

Partha Chatterjee, Politique des gouvernés. Réflexions sur la politique populaire dans la majeure partie du monde (traduit de l'anglais (Inde, États-Unis) par Christian Jaquet), Paris, Amsterdam, 2009 [2004], p. 125-129. tiques de puissances tierces, d'exigences coloniales propres de « rattraper son retard », etc. - les colonies pouvaient être de façon compensatoire mises au service du rétablissement de l'équilibre des puissances internationales - lequel était en fait essentiellement intraeuropéen. Une telle idée est typiquement «impérialiste » et étrangère à une pensée « colonialiste », qui considère qu'on a « acquis » les colonies ou qu' on vous les a «confiées» durablement. L'impérialisme est planifié et exécuté par les chancelleries d'État, les

ministères des Affaires étrangères ou de la Guerre, le colonialisme l'est par des autorités coloniales ou par des «men on the spot» (John S. Galbraith). On peut certes appréhender une telle Weltpolitik du point de vue de l'histoire événementielle ; il ne faut néanmoins pas perdre de vue qu'elle a (1) pour arrière-plan la formation d'un système étatique mondial et (2) pour cadre un lent développement de structures inégalitaires dans les échanges économiques entre les différentes parties de la planète. 
$\mathrm{L}^{\prime}$ « impérialisme » et le « colonialisme » ne sont donc pas identiques. À plus d'un égard, l' «impérialisme » est un concept plus global dont le «colonialisme » n'est qu'une variété spécifique, comme cela peut se vérifier aisément en ce qui concerne l'Empire britannique à partir des années 1780. Mais comme l'« impérialisme» inclut la possibilité d'appréhender des intérêts à l'échelle mondiale et, à partir d'appuis informels, de pénétrer de grands espaces économiques selon un mode capitaliste, on hésitera à appliquer ce concept pendant la période moderne où ces capacités n'existaient pas : c'est donc avec prudence que l'on parlera de l'« impérialisme espagnol ». Seuls la Grande-Bretagne et les États-Unis ont atteint le statut de puissances impérialistes au sens plein du terme - les États-Unis étant assurément un cas d'impérialisme sans empire colonial.

Selon les périodes, la France, l'Allemagne, la Russie (puis l'Union soviétique) et le Japon ont été impérialistes dans un sens restreint : soit ces pays n'ont pu se maintenir à l'échelle mondiale de façon durable, soit, comme l'Union soviétique à l'apogée de sa puissance militaire, ils ne disposaient pas d'une puissance économique leur permettant de pénétrer des économies lointaines. Les empires coloniaux sans impérialisme ont été la règle lors de la phase d'expansion que l'Europe a connue pendant la période moderne; seuls les Pays-Bas au XVII ${ }^{\mathrm{e}}$ siècle peuvent éventuellement être considérés comme une exception. Mais aux XIX ${ }^{\mathrm{e}}$ et $\mathrm{Xx}^{\mathrm{e}}$ siècles, ce sont justement les Pays-Bas qui illustrent parfaitement ce cas. Pendant l'Entre-deux-guerres, les Pays-Bas étaient sans aucun doute la troisième puissance coloniale derrière la Grande-Bretagne et la France. Mais dans le même temps, ils n'étaient, à aucun égard ou presque, une puissance impérialiste : ils n'avaient ni l'ambition ni les moyens nécessaires pour mener une Weltpolitik, et ils ne disposaient pas de la possibilité de s'imposer aucunement de façon « informelle » au-delà de leur colonie indonésienne?

Traduit de l'allemand par Thierry Carpent

1. Philip D. Curtin, « The Black Experience of Colonialism and Imperialism », dans Sidney W. Mintz (dir.), Slavery, Colonialism, and Racism, New York, Norton, 1974, p. 23.

2. Cf. Michael Winter, Egyptian Society under Ottoman Rule 1517-1798, Londres / New York, Routledge, 1992, p. 30. 


\section{Labyrinthe, $n^{\circ} 35$}

3. Edward W. Said, Culture and Imperialism, Londres, Knopf, 1993, p. 8.

4. «A spiritual yoke » : Philip Mason, Patterns of Dominance, Londres / New York, Oxford University Press, 1970, p. 274.

5. Au chapitre précédent [N.d.F.].

6. Cf. les commentaires, présentant à la fois un résumé et une critique, de Robert J. Hind, «The Internal Colonial Concept », Comparative Studies in Society and History 26/1984, p. 543-68. Parler de "périphérie intérieure » aurait davantage de sens : cf. Hans-Heinrich Nolte (dir.), Internal Peripheries in European History, Göttingen, Muster-Schmidt, 1991.

7. Les historiens anglais Ronald E. Robinson et John A. Gallagher ont donné les impulsions fondamentales sur la question. Cf. particulièrement Wm. Roger Louis (dir.), Imperialism: The Robinson and Gallagher Controversy, New York 1976 ; R.E. Robinson, « The Excentric Idea of Empire - with or without imperialism », dans Wolfgang J. Mommsen, Jürgen Osterhammel (dir.), Imperialism and After : Continuities and Discontinuities, Londres / Boston, German Historical Institute / Allen \& Unwin, 1986, p. 267-89. Cf. également les importantes réflexions, qui approfondissent la question, de Michael W. Doyle, Empires, Ithaca / Londres, Cornell University Press, 1986, p. 19-47.

8. Cf. les ouvrages, complémentaires, de Ramon H. Myers/Mark R. Peattie (dir.), The Japanese Colonial Empire, 1895-1945, Princeton, Princeton University Press, 1984, et de Peter Duus et alii (dir.), The Japanese Informal Empire in China, 1895-1937, Princeton, Princeton University Press, 1989. William G. Beasley, Japanese Imperialism 1894-1945, Oxford, Clarendon Press, 1987, donne un excellent aperçu.

9. Cf. Jurrien van Goor, «Imperialisme in de marge ? d dans id. (dir.), Imperialisme in de marge :

De afronding van Nederlands-Indie, Utrecht, HES, 1986, p. 9. 